\title{
El impacto de las redes sociales en la promoción de lectura y venta de libros en Latinoamérica entre 2010-2020
}

\section{The impact of social media in the promotion of reading and book sales in Latin America between 2010-2020}

\author{
Camila Giuria Farias \\ Pontificia Universidad Católica del Perú \\ a20205815@pucp.edu.pe
}

\begin{abstract}
Resumen
Las subcomunidades de booktube y bookstagram en redes sociales han tenido un gran impacto en los hábitos de lectura y negocios relacionados a los libros en Latinoamérica en la última década. El presente trabajo estudia la influencia de las redes sociales YouTube e Instagram en la promoción de lectura y venta de libros, al igual que las causas y consecuencias de este fenómeno. Las facilidades de comunicación de cada red social debido a las capacidades multimedia de sus plataformas determinan el éxito de cada una en diferentes aspectos, ya que Instagram resulta ideal para marketing de ventas online, y YouTube cumple con propósitos persuasivos que consiguen promover hábitos de lectura. Este artículo concluye que, de las dos plataformas presentadas, YouTube y la comunidad de booktube han tenido una gran influencia en la promoción de la lectura debido a la facilidad con la que se puede crear un lazo de conexión; e Instagram tuvo mayor éxito en las ventas online y ferias del libro, por la facilidad para enviar un mensaje conciso.
\end{abstract}

Palabras claves: booktube; bookstagram; redes sociales; promoción de lectura; venta de libros

\begin{abstract}
The booktube and bookstagram subcommunities on social networks have had a great impact on reading habits and book-related businesses in Latin America in the last decade. This paper studies the influence of the social networks YouTube and Instagram on the promotion of reading and book sales, as well as the causes and consequences of this phenomenon. The communication facilities of each social network due to the multimedia capabilities of their platforms determine the success of each one in different aspects, since Instagram is ideal for online sales marketing, and YouTube fulfills persuasive purposes that manage to promote reading habits. This article concludes that, of the two platforms presented, YouTube and the booktube community have had a great influence in the promotion of reading due to the ease with which a connection link can be created; and Instagram had a greater success in online sales and book fairs, due to the ease of sending a concise message.
\end{abstract}

Keywords: booktube; bookstagram; social media; reading promotion; book sales 
El impacto de las redes sociales en la promoción de lectura y venta de libros...

\section{Introducción}

'Los jóvenes de ahora no leen': Una frase reiterada constantemente por estudios que determinan a la lectura por placer como una práctica únicamente realizada en últimas instancias de aburrimiento por la actual juventud (Vílchez, 2003). Sin embargo, quienes sostienen la posición previamente expresada suelen basarse en datos que no son recientes y no toman en cuenta el impacto de las redes sociales, las cuales actualmente influencian el día a día de la mayoría en diversos aspectos. Como menciona la revista virtual Notimérica (2018): «Con el desarrollo de plataformas multimedia, como las redes sociales [...], los hábitos de lectura han cambiado en la última década». Cabe mencionar que en Latinoamérica es donde se ha notado un gran impacto en la tasa de jóvenes lectores, ya que en los países del continente solía haber un promedio de lectores mínimo el cual ha ido aumentando progresivamente, siendo este un incremento en la alfabetización digital y estimulación de las competencias tanto lingüísticas como comunicativas (Levano-Francia y otros, 2019).

Los estudios e investigaciones que respectan al impacto de las redes sociales en el mundo lector son relativamente recientes, $y$ son abordados principalmente desde la perspectiva de la promoción de la lectura y la promoción de la venta de libros; aspectos que se ven correlacionados, pero tienen una gran distinción en las investigaciones. También se crea una gran distinción entre las redes sociales en las que la interacción lectora sucede, debido a que cada una funciona en una modalidad diferente y tiene un impacto diferente. Por un lado, se ha estudiado la estrategia comunicativa que tienen estos promotores de lectura hispanohablantes en la red social YouTube, la cual consta de videos y otros elementos multimedia, y explicando el porqué de su éxito en promover la lectura de los libros que recomiendan (Pretel, Frutos y Sánchez-Valle, 2019); mientras que por otro lado se analiza el impacto de esta misma red social desde un punto de vista más monetario y con respecto a la venta de libros e industria editorial (Tomasena, 2019).

Por consiguiente, el presente trabajo busca estudiar el impacto de las redes sociales en los hábitos de lectura y promoción de venta de libros, centrado principalmente en las redes sociales de Instagram y YouTube y responder a la siguiente pregunta: ¿De qué manera las redes sociales han promocionado la lectura y venta de libros en Latinoamérica entre el 2010-2020? En el tiempo actual, este tema resulta relevante debido a que las redes sociales ocupan gran parte de la vida de un ciudadano promedio, y con el paso del tiempo se está viendo como diferentes

72 Lengua y Sociedad. Revista de Lingüística Teórica y Aplicada 
El impacto de las redes sociales en la promoción de lectura y venta de libros...

Camila Giuria Farias

plataformas afectan de manera única comunidades, como la lectora, y no existen trabajos que analizen a detalle el impacto dedicándose a más de una red social y más de un aspecto simultáneamente.

Para cumplir con dicho propósito se dividirá el trabajo en, primeramente, el impacto de Bookstagram y Booktube en la promoción de la lectura; y, luego, se verá como la venta de libros es motivada por estas mismas redes, al haber promocionado principalmente las ventas online y la asistencia a ferias del libro.

\section{Aspectos teórico-conceptuales}

\subsection{Importancia de la lectura}

La comprensión lectora es una capacidad esencial para el desarrollo de los seres humanos, con la que se decodifica y comprende un complejo texto para captar un mensaje, y es desarrollada por medio de la constante lectura (Ortega, 2015). Es importante para el desarrollo del pensamiento crítico, aumento de la memoria y establecimiento de lazos de empatía; por lo que el declive en su práctica resulta preocupante.

La lectura es una actividad famosa por sus beneficios y durabilidad a través del tiempo, pero en ciertas áreas del mundo resulta inaccesible debido a altas tasas de analfabetismo o la ausencia de bibliotecas o espacios para conseguir material de lectura. Inclusive, cuando se encuentra en un área con accesibilidad a todo lo antes mencionado, se suele considerar a la lectura como una tarea y no una actividad de recreación, en consecuencia se ha dejado de practicar y reemplazado con aficiones relacionadas a la tecnología como videojuegos o fotografía (V por Vanguardia, 2019).

Ante todo lo previamente mencionado, actualmente existen promotores de lectura que buscan diferentes plataformas tanto físicas como digitales con el propósito de crear más lectores al compartir su dedicación por los libros, e incluso llegan a ser auspiciados por editoriales o tiendas para promover la venta de ciertos libros.

\subsection{YouTube e Instagram y los influencers}

Tanto YouTube como Instagram son plataformas digitales reconocidas como redes sociales, las cuales fueron creadas entre los años 2005 y 2010, y tomaron mayor relevancia a partir del 2010 hasta la actualidad. La primera es una plataforma en la 
El impacto de las redes sociales en la promoción de lectura y venta de libros... Camila Giuria Farias

que cualquier usuario registrado puede subir sus propios videos, y si el contenido es del gusto de otros usuarios, estos se pueden suscribir a su canal para recibir notificaciones sobre futuros videos. De la misma manera, Instagram es una red social en la que los usuarios deben crear perfiles y en ellos pueden elegir subir fotos o videos cortos que se mantendrán permanentemente en la plataforma, o pueden subir «historias» las cuales son igualmente fotos o videos, pero estos se eliminan de la plataforma luego de 24 horas de su publicación. También se puede seguir a un usuario para estar pendiente de el contenido que subirá a futuro.

La definición de la palabra influencer es la traducción literal del inglés al español: hombre (o persona) de influencia; y en el contexto digital ejercen dicha influencia en el público que los sigue. Estas redes sociales han sido y siguen siendo utilizadas para la mercadotecnia influyente, la cual se sostiene de la imagen de una personalidad famosa en redes sociales que ha creado un lazo de confianza con su público, con el propósito de publicitar algún tipo de producto o servicio con anuncios pagados a este influencer. Para la publicidad de algún producto hoy en día resulta inconcebible que la campaña de marketing no contenga algún tipo de promoción en redes sociales colaborando con influencers reconocidos en el nicho de mercado del producto; debido a que las redes sociales se han vuelto casi una segunda naturaleza para cualquier aspecto de la vida actual.

Cabe mencionar que no todo influencer ocupa su efecto sobre sus seguidores con fines de lucro, ya que existen personalidades las cuales ejercen una influencia sin generar ingresos debido a esto, como en muchos casos los influencers lectores que promueven la lectura sin estar asociados a alguna empresa o asociación, simplemente comparten su pasión y tiene un público que confía en ellos.

\section{Metodología}

El presente artículo es un estudio descriptivo el cual detalla las características de las subcomunidades lectoras en redes sociales y el impacto que han tenido en países latinoamericanos, sosteniendo los datos en fuentes de información especializadas en el tema. Para analizar estos datos, primero se presentarán las carácterísticas de las dos plataformas virtuales que serán estudiadas (YouTube e Instagram), y a continuación se presentarán las variables de la promoción de la lectura y la venta de libros/ferias del libro. Entonces, en cada variable se expondrá detalladamente la relación que tiene con las subcomunidades en cada red social, para finalmente concluir que plataforma tuvo mayor éxito con respecto a cada variable.

74 Lengua y Sociedad. Revista de Lingüística Teórica y Aplicada 


\section{Análisis}

\subsection{Promoción de la lectura en redes sociales}

Las redes sociales han tomado gran importancia durante la última década, llegando a convertirse en un medio de comunicación esencial, un espacio para aprender, trabajar y compartir intereses personales; básicamente alfabetización mediática e informacional. Con el establecimiento de ciertas redes sociales, se reconoce la creación de subcomunidades en estas redes donde los usuarios pueden compartir intereses más particulares, como por ejemplo la comunidad de Bookstagram en la red social Instagram, que Scherer afirma combina las pasiones por la lectura y la fotografía (2016, p. 1). En estas comunidades ha surgido la necesidad de compartir contenido relacionado a libros ya que «la lectura se vuelve una práctica que no solo necesita ser vivida sino también compartida y socializada. Este es el principio fundamental de la comunidad booktuber» (Maestre Santamaría, 2019).

Las figuras que ejercen influencia sobre esta comunidad son comúnmente referidas como influencers literarios y, principalmente, comparten su opinión sobre libros en forma de reseñas con el mundo, y según Pretel, De Frutos y SánchezValle son mayormente mujeres jóvenes que producen y editan su contenido individualmente con temáticas variadas (2019, p. 12).

En Bookstagram, se suele encontrar fotos de libros con otros elementos literarios (como tazas, separadores, adornos o velas), y en la descripción de la foto se encuentra un pequeño texto que suele describir el propósito de la foto. Por ejemplo, en la descripción se escribe que esa foto es un bookhaul, o contenido sobre los libros comprados u obtenidos durante el mes de la publicación (Pretel, Frutos y Sánchez-Valle, 2019), y en la imagen se visualiza una pila de libros acomodados. Las fotos ya mencionadas suelen ser llamativas al público por su edición, o el orden de los elementos en la foto que les da un sentimiento de orden o productividad.

El éxito de este medio de promoción de lectura radica en la nueva generación $\mathrm{Z}$, la cual suele seguir tendencias o acciones que ven de sus influencers favoritos y que consideren beneficiosas; pero ante esto surge la duda de si se está verdaderamente promoviendo el acto de leer o el estilo de vida y «estética» de un lector, que vendría acompañado con el consumismo basado en la compra y almacenamiento de libros sin en verdad leerlos. Esta idea es respaldada con la naturaleza de la red social Instagram, la cual suele ser utilizada como un medio de anuncios publicitarios o la publicación de fotos que muestran un estilo de vida «perfecto»e «inalcanzable». Además, la colaboración entre editoriales o tiendas de libros con 
El impacto de las redes sociales en la promoción de lectura y venta de libros... Camila Giuria Farias

estos influencers han colaborado con prescindir de la promoción de la lectura en la red social y lo ha reemplazado por una carrera de quién puede tener más libros en sus estanterías.

Por ello, el bookstagrammer Nils Kückmeister afirma: «Es mejor adentrarse en la literatura que no hacerlo... Si puedes combinar la creatividad con la lectura de libros y el debate sobre ellos, ¿por qué no?»; aludiendo que, a pesar de no ser una red social perfecta, es mejor adentrarse a ella sin el propósito explícito de promover la lectura, a no hacerlo por completo.

En síntesis, la red social Instagram dio lugar a la comunidad de Bookstagram en la que miles de lectores conviven compartiendo fotos de sus lecturas acompañadas de recomendaciones o reseñas, y estos lectores se han convertido en una influencia para la sociedad logrando promover la lectura como hábito para la juventud actual, pero sin tenerlo como objetivo también crearon una comunidad de consumismo donde se suele perder de vista el propósito original, leer; y es reemplazado con la compra y venta de libros.

De la misma forma que se denomina Bookstagram a la comunidad de lectores en la red social Instagram, se le llama Booktube a la comunidad lectora en YouTube. Se considera incluso que Booktube es una comunidad más antigua y establecida que Bookstagram al haber estado más años presente como influencia en el internet. En esta plataforma también se comparten opiniones y reseñas sobre libros, pero la mayor diferencia es la presencia de diferentes medios audiovisuales, al igual que un mayor tiempo para explayar sus ideas.

Ahora, ¿por qué son tan exitosos estos canales si su contenido no está siendo manejado por un profesional en educación o promoción de lectura? La respuesta resulta sencilla para la juventud actual, se reconoce entonces que el principal motivo por el que estos influencers están triunfando es que se refieren a los libros de una manera muy familiar, como una parte de ellos mismos, así como añaden tonos humorísticos acompañados de expresiones corporales y frases comunes que hace sentir al espectador joven conectado (López, 2017). Como mencionan Pretel, De Frutos y Sánchez-Valle, estos contenidos con tonos humorísticos los cuales no solo se ciñen a la literatura, sino que se adaptan a la plataforma de YouTube y la nueva generación (2019, p. 17). Los booktubers son capaces de fomentar la lectura al llegar al espectador más profunda y efectivamente que un profesor con herramientas como la edición de los videos o incluso el léxico emplean (López, 2017, p. 966). Esta idea que sustenta la acogida de los booktubers, debido a su forma de hablar y estilo identificable, ha sido reforzada con el paso de los años:

76 Lengua y Sociedad. Revista de Lingüística Teórica y Aplicada 
El impacto de las redes sociales en la promoción de lectura y venta de libros...

Camila Giuria Farias

Su lenguaje basado en tono directo, sencillo, honesto y llano y siempre apasionado, se acerca e impacta al target de consumo que tiene la red social. Este tono, junto a la autenticidad que reflejan los comentarios que vuelcan sobre las obras literarias, seguramente les haga ganar credibilidad en su discurso [...] (Pretel, Frutos y Sánchez-Valle, 2019, p. 17)

Además, como menciona Maestre (2019, 17), las particularidades de este lenguaje posibilita un discurso repetitivo que se relaciona directamente con su opinión de cierto libro y genera una respuesta e interacción de la comunidad espectadora online. Sintetizando, la red social de YouTube ha tenido un gran impacto en la promoción de la lectura en la comunidad hispanohablante, e incluso se podría considerar que ha tenido mayor éxito en promover la lectura que la red social Instagram como menciona un $62.1 \%$ de encuestados que mencionan que Youtube es su fuente de recomendaciones de libros, mientras que tan solo un $7.7 \%$ prefiere consumir información y entretenimineot de otros medios virtuales com Instagram (Rodrigo, 2017); esto se debe a que en YouTube los creadores y creadoras de contenido tienen acceso a más medios para conectar con la audiencia como su apariencia en la pantalla, el tono que utilizan al hablar o la edición de los elementos multimedia de la videograbación final. A diferencia de una publicación de una imagen con texto en bookstagram, por medio de booktube el influencer pudo encontrar un «método de persuasión» para promover la lectura que no se había visualizado en otras redes sociales. Así, se concluye que, aunque ambas redes sociales tuvieron un mismo propósito, una red social lo pudo lograr más plenamente debido a las características de la misma plataforma, mientras que la otra influyó mayormente en la promoción de la venta o consumo de libros.

\subsection{Venta de libros motivada por redes sociales}

Como se mencionó en el subtema anterior, las comunidades de Booktube y Bookstagram promovieron en gran manera la venta de libros en sus plataformas, aunque su propósito inicial haya sido promocionar la lectura, por lo que no resulta sorprendente que diversas empresas relacionadas al universo literario aprovecharan de estas redes sociales con fines lucrativos. Menciona Alberto Mejía (2020): «Las editoriales buscan alternativas para empujar la venta de libros y emplean canales de comunicación más auténticos. Los booktubers son sus aliados para estas acciones» (Alberto Mejía, citado por Pastor, 2020). 
El impacto de las redes sociales en la promoción de lectura y venta de libros...

Camila Giuria Farias

Por un lado, las ferias del libro se han visto impactadas por el fenómeno del internet, ya que ha aumentado sustancialmente la asistencia a estos eventos culturales a partir de sus promociones en redes sociales. En el caso partículas de la Feria Internacional del Libro de Lima, el 2018 fue el primer año que tuvo un evento llamado Encuentro Internacional de Booktubers (que a partir del 2019 fue llamado Encuentro Internacional de Booktubers y Bookstagrammers); y a partir de ello se ha visto un incremento en la cantidad de asistentes, la frecuencia y duración de sus visitas, y la compra en general de ejemplares dentro de la feria. Incluso ante la virtualidad forzada por la pandemia del nuevo Covid-19 se ha encontrado que, gracias a la promoción y presencia de estos influencers no exclusivamente peruanos se pudo lograr: 869 mil visualizaciones en los eventos de la feria alcanzando a aproximadamente 3.9 millones de personas a nivel internacional, y una venta de más de 1.2 millones de soles peruanos (Feria Internacional del Libro de Lima, 2020). En la edición de la feria del libro de Lima del 2019 incluso se reconoce tres géneros principales que han sido los más vendido: Crónica/Periodismo, Ensayo (principalmente de filosofía y política), y desarrollo personal; así se resalta como estas ventas de libros representan una oportunidad al público de crecimiento intelectual de distintas temáticas y llegan a formar parte de la educación de los países latinoamericanos al relacionarse con el desarrollo personal con respecto a cultura, literatura e historia (La República, 2019). También, como menciona Redacción Cultura (2017), de Prensa Libre, no solo se lee no-ficción en la región debido a que el auge del cine ha generado un aumento drástico en la lectura de géneros de fantasía o ficción en general sobre todo entre la juventud (Estos son los libros más leídos de Latinoamérica, 2017).

Basta con un aspecto tan pequeño como un video de «Recomendaciones para comprar en la feria del libro» o «Novedades para la FIL de este año», el creador de contenido en las redes sociales promueve la asistencia a estos eventos, y muchas veces lo hacen indirectamente ya que no tienen ningún tipo de patrocinio por estas ferias.

En el caso de las ventas virtuales, también conocido como el e-commerce de libros, se ha visto una abrumante presencia de motivación a la compra en la red social Instagram aparentemente relacionada con la brevedad con la que se puede entregar el mensaje al espectador (Fernández, 2018). Se reconoce que es más sencillo promover la compra usando un código cupón en un texto decorado con menos de 10 palabras que con un video de 5 minutos. Ante esto, la red social Instagram se ha convertido en una gran plataforma de compra y venta de

$78 \quad$ Lengua y Sociedad. Revista de Lingüística Teórica y Aplicada 
El impacto de las redes sociales en la promoción de lectura y venta de libros...

Camila Giuria Farias

diferentes objetos, y la comunidad de libros no se ha quedado atrás con ello. En las redes sociales de librerías latinoamericanas como Íbero Librerías en Perú o Ghandi en México, se ve un intento por conectar con un público que impulsa la venta en sus medios virtuales, sobre todo ahora que comprar online es la forma más segura en el aspecto sanitario. Si bien es cierto muchas de estas editoriales o tiendas emplean a un influencer para promocionarse, por ejemplo dándoles cupones de descuento exclusivos para sus seguidores, se puede evidenciar cierta separación de estos bookstagrammers, ya que se centra en crear su propia imagen en redes sociales.

Entonces, aunque la pandemia ha promovido las ventas online, se puede denotar que los vendedores o proveedores de libros han estado trabajando en su imagen en redes sociales como estrategia comunicativa para conectar con un público con el que potencialmente creen una relación exclusiva y la compra de un libro online se vuelva un hecho repetitivo.

Ante todo lo mencionado, se establece una relación entre el aumento de asistencia a las ferias del libro en Latinoamérica y las ventas online con las redes sociales. A pesar de que el impacto es muy similar en lo que respecta a las ferias, se reconoce que Instagram ha tenido una mayor importancia en la venta de libros debido a la naturaleza de la plataforma y la facilidad con la que el mensaje puede ser enviado en comparación a Youtube. Además, en las ferias del libro la presencia de un influencer, ya sea booktuber o bookstagrammer, tiene mayor importancia que en las ventas online debido a que la asistencia a los eventos se ve relacionada a los personajes que se presenten en ellos, mientras que en las ventas las librerías o tiendas están creando sus propias personalidades al estar tomando importancia con diferentes estrategias de relacionamiento con el público y comunicación eficaz. Así, se puede ver como Instagram logró el propósito de venta de libros motivada por redes sociales mejor que YouTube.

\section{Conclusiones}

En síntesis, esta investigación concluye que las redes sociales han tenido un gran impacto en la comunidad de lectores en Latinoamérica entre el 2010 y el 2020 en los aspectos de promoción de la lectura y venta de libros.

De las dos plataformas estudiadas, Youtube y la comunidad de Booktube han tenido una gran influencia en la promoción de la lectura debido a la facilidad con la que se puede crear un lazo de conexión entre los espectadores por medio 
El impacto de las redes sociales en la promoción de lectura y venta de libros...

Camila Giuria Farias

de diversos medios de comunicación. Como menciona Marilia Pastor (2020), los booktubers recomiendan lecturas por medio de frescos y divertidos videos de YouTube y, de esta manera, cosechan seguidores y también capturan nuevos lectores. La facilidad comunicativa es entonces el factor más importante en este aspecto para esta primera red social, mientras que Instagram no tuvo tanto éxito debido a la desviación de la comunidad de Bookstagram de su propósito original que la llevó a crear involuntariamente una cultura de consumismo.

Entonces, Instagram tuvo mayor importancia en el segundo aspecto de las ventas online y ferias del libro, también guiada por la misma plataforma y la facilidad de enviar un mensaje conciso. Además, se ha visto como en la promoción de la lectura por medio de redes sociales requieren la presencia de una figura amigable en forma de influencer que conecte con los espectadores y los entretenga de cierta manera que persuada a la lectura, mientras que para la venta de libros las mismas tiendas o editoriales van creando sus propias personalidades en las redes sociales (sobre todo Instagram) creando una relación de exclusividad y lealtad con el público. Además, se evidencia como las ventas de libros no necesariamente representan solo a una cultura consumista, sino también a el respaldo de la educación de la región en vista de que la mayoría de géneros vendidos en ferias del libro son para el enriquecimiento intelectual.

Para finalizar, cabe mencionar que, aunque las redes sociales han impactado en gran manera en diferentes aspectos de la vida en la región latinoamericana debido a que en promedio los minutos diarios empleados en redes sociales en la región alcanzan 212 minutos (Duarte, 2019) no significa que haya existido un crecimiento extremo de lectores. Sí es cierto que han aumentado los libros leídos por año, ahora ferias del libro internacionales como la de Guadalajara y Buenos Aires tienen un gran porte en el extranjero, y las competencias personales de los lectores relacionadas al aprendizaje se han visto estimuladas; pero aún así existen distintas limitaciones que sufre la comunidad lectora en Latinoamérica, las cuales no se presentan en otras regiones: por ejemplo, es más difícil que un bookstagrammer o booktuber tenga una relación cercana y duradera con una editorial al ser hispanohablante, ya que suelen ser utilizados para promociones cortas o lanzamientos específicos (Tomasena, 2019); o se complica para un autor latinoamericano publicar sus libros en otras regiones, que sean traducidos a diferentes idiomas o incluso se adapten a películas y series debido a una barrera construida por prejuicios establecidos contra la región.

8o Lengua y Sociedad. Revista de Lingüística Teórica y Aplicada 
El impacto de las redes sociales en la promoción de lectura y venta de libros...

Camila Giuria Farias

En un caso más específico del Perú, las comunidades de booktube y bookstagram están creciendo lentamente, pero su impacto se evidencia en el éxito de las ferias del libro, así como la creación del Encuentro Internacional de Booktubers y Bookstagrammers, y los esfuerzos por diversas entidades en el país para promover la lectura en redes sociales, como la creación de una plataforma virtual de la Biblioteca Nacional del Perú para que el público general pueda acceder a contenido de lectura.

Como última recomendación, se anima al público poder consumir contenido de libros originalmente latinoamericano, que cada día es más sencillo debido a la presencia de redes sociales que nos facilitan la recomendación de títulos y medios para leer, que no solo significa el apoyo a influencers virtuales y autores, sino que impulsa la educación por medios virtuales, ejercitando nuestro cerebro y distintas habilidades relacionadas a la lingüística, narrativa y crítica literaria; además de fomentar la alfabetización mediática y digital debido al aprendizaje del uso de redes sociales y medios virtuales.

\section{Referencias bibliográficas}

Duarte, F. (2019, 9 de setiembre). Los países en los que la gente pasa más tiempo en las redes sociales (y los líderes en América Latina). BBC News. https://www. bbc.com/mundo/noticias-49634612

Feria Internacional del Libro de Lima. (2020). https://www.fillima.com.pe

Fernández, M. (2018). La influencia de la comunidad bookstagram en el lanzamiento y promoción de un libro (Trabajo de maestría). Universidad de Almería, España.

La República. (2019, 5 de agosto). Por cada género: los libros más vendidos de la FIL Lima 2019. La República. https:/larepublica.pe/cultural/2019/08/05/ fil-lima-2019-por-cada-genero-los-libros-mas-vendidos-de-la-feria/

Levano-Francia, L., Sanchez Diaz, S., Guillén-Aparicio, P., Tello-Cabello, S., Herrera-Paico, N. y Collantes-Inga, Z. (2019). Competencias digitales y educación. Propósitos y Representaciones, 7(2), 569-588.

López, M. L. (2017). Booktubers y Literatura. Revista Publicando, 4(13[2]), 936-974. https://revistapublicando.org/revista/index.php/crv/article/view/980

Maestre Santamaría, V. (2019). Booktubers, prácticas de lectura en el siglo XXI y convergencia digital [Tesis de licenciatura, Pontificia Universidad Javeriana]. 
El impacto de las redes sociales en la promoción de lectura y venta de libros...

Repositorio Institucional de la Pontificia Universidad Javeriana. https://repository.javeriana.edu.co/handle/10554/47243

Notimérica. (2018, 11 de noviembre). Día internacional del libro: ¿Qué país lee más en Iberoamérica? Notimérica. https://www.notimerica.com/sociedad/noticia-dia-internacional-libro-pais-lee-mas-iberoamerica-20180423111031.html

Ortega, F. J. (2015). La importancia de la lectura y de las nuevas tecnologías en el aprendizaje del español para inmigrantes. Investigaciones sobre Lectura, 3 , 123-133.

Pastor, M. (2020, 26 de agosto). Los booktubers: El elemento clave para convertir al Perú en un país de lectores. RPP Noticias. https://rpp.pe/cultura/literatura/ fil-lima-2020-booktubers-el-elemento-clave-para-convertir-al-peru-en-unpais-de-lectores-noticia-1288353

Pretel, M., Frutos, B. D. y Sánchez-Valle, M. (2019). El influencer literario a través de YouTube: análisis de la estrategia comunicativa. Obra Digital, 17, 9-18.

Redacción Cultura. (2017, 12 de marzo). Estos son los libros más leídos de Latinoamérica. Prensa Libre. https://www.prensalibre.com/vida/escenario/ estos-son-los-libros-mas-leidos-en-america

Rodrigo, I. (2017, 26 de abril). ¡Los resultados más esperados! Encuestas a los espectadores de Léeme. Léeme. https://leemetv.com/habitos-lectores

Scherer, F. (2016, 6 de mayo). $42^{\circ}$ Feria del libro / Último fin de semana. Libros en la red: La literatura juvenil ahora se juega en Instagram. La Nación. https:// www.lanacion.com.ar/cultura/lectores-en-la-red-la-ultima-evolucion-de-la-literatura-juvenil-se-juega-en-instagram-nid1895915/

Tomasena, J. (2019). Negotiating collaborations: Booktubers, the publishing industry and Youtube's ecosystem. SAGE Journals, 5(4), 1-12.

V por Vanguardia. (2019, 20 de junio). Los hobbies más populares actualmente. Vanguardia: https://vanguardia.com.mx/articulo/los-hobbies-mas-popularesactualmente

Vílchez, C. (2003). Hábitos de lectura de los adolescentes peruanos: nuevas perspectivas. Revista Interamericana de Bibliotecología, 26(2), 1-13.

\section{Trayectoria académica de la autora}

Camila Giuria Farias es graduada del programa de Diploma del Bachillerato Internacional, y actualmente es estudiante de Lingüística y Literatura en la Pontificia Universidad Católica del Perú. Sus áreas de interés son las redes sociales, la lectura en Latinoamérica, y problemáticas que conciernen a la juventud.

82 Lengua y Sociedad. Revista de Lingüística Teórica y Aplicada 